\title{
Pleural procedures: training the trainee
}

\author{
Authors: Zhe Wu and Owais Kadwani
}

\section{Aims}

Two respiratory registrars with level 1 ultrasound competency aimed to set up a service to provide supervised pleural procedures training for trainees at a district general hospital.

\section{Methods}

An initial online survey was sent out via email to gauge trainees' interest, their level of previous experience in pleural procedures and their curriculum requirements, allowing us to target our programme. Following this, trainees were asked to provide 1-week slots where they would be available to be trained and were allocated weekly slots on a 'pleural rota' that gave them priority over any pleural procedures. During the programme, we used the smartphone-based application 'WhatsApp' for quicker responses when the allocated trainee was unavailable. For every procedure, we consented the patient, performed a bedside ultrasound scan and supervised a trainee performing a pleural procedure. The level of supervision varied depending on the experience of the trainee. We repeated the survey after a 10 -week period in order to gauge results.

\section{Results}

Our initial survey, which was conducted in March to April 2017, had 24 responses. Two trainees had not performed any pleural procedures and 14 had only performed between one to five procedures. Twenty-five percent had no confidence to perform a pleural aspirate unsupervised while $50 \%$ had no confidence to perform a chest drain unsupervised. Furthermore, two-thirds of trainees found it extremely difficult to obtain competency sign-off (directly observed practical procedure forms or direct observation of procedural skills (DOPS)). Following our 10-week training programme, 17 trainees responded to our feedback survey, 12 of whom obtained procedures through the programme. Seven trainees managed three or more supervised procedures and 10 trainees obtained DOPS forms for all procedures. Seventy per cent of respondents felt more confident performing pleural aspirates and $55 \%$ felt more confident in performing chest drains following the programme.

\section{Conclusion}

The programme has led to most trainees achieving their required assessments for their curriculum training while also increasing the level of confidence with pleural procedures. The use of phonebased technology allowed for quicker response time and meant each opportunity could be advertised to all trainees on the rota. Written feedback has shown the programme to be a success with most trainees asking the rota to continue for the new yearly intake of doctors to our hospital. We have since delivered a pleural training session as part of induction to all core medical trainees who joined the trust in August 2017.

\section{Conflict of interest statement}

There are no conflicts of interest to declare. 